IMECE2003-41845

\title{
AN EFFICIENT LANE CHANGE MANEUVER FOR PLATOONS OF VEHICLES IN AN AUTOMATED HIGHWAY SYSTEM
}

\author{
Xiaotian Sun, Roberto Horowitz \\ Department of Mechanical Engineering \\ University of California at Berkeley \\ Berkeley, California 94720-1742 \\ Email: \{sunx, horowitz\}@me.berkeley.edu
}

\author{
Chin-Woo Tan \\ California PATH, Headquarters \\ University of California, Berkeley \\ Richmond Field Station, Bldg. 452 \\ 1357 South 46th Street \\ Richmond, California 94804-4698 \\ Email: tan@robotics . eecs . berkeley.edu
}

\begin{abstract}
The current lane change maneuver for vehicles in a platoon under the California PATH automated highway system (AHS) architecture is inefficient, because the follower has to split from the rest of the platoon before making a lane change. In this paper, we propose to add a lane change within platoons maneuver that allows a follower to change lanes and be inserted into another platoon directly without splitting either platoon. This maneuver is performed by aligning and locking the longitudinal positions of the two platoons in adjacent lanes. The estimated improvement in the AHS utilization, in term of the space-time, is approximately $4342 \mathrm{~m} \cdot \mathrm{s}$. The longitudinal controller for the lane changing follower is designed and proved to maintain the string stability of the platoons. The leader law is modified for the common leader of the two locked platoons. An intra-platoon spacing adjustment procedure is also designed for the purpose of the proposed maneuver.
\end{abstract}

\section{INTRODUCTION}

In the normal operation mode of the California PATH (Partners for Advanced Transit and Highways) automated highway system (AHS) architecture [1,2], the control system is built on a hierarchy of five layers, which from the top to the bottom, as shown in Figure 1, are network, link, coordination, regulation, and physical layers.

The network and link layers reside on the road side. The

*Address all correspondences to this author.

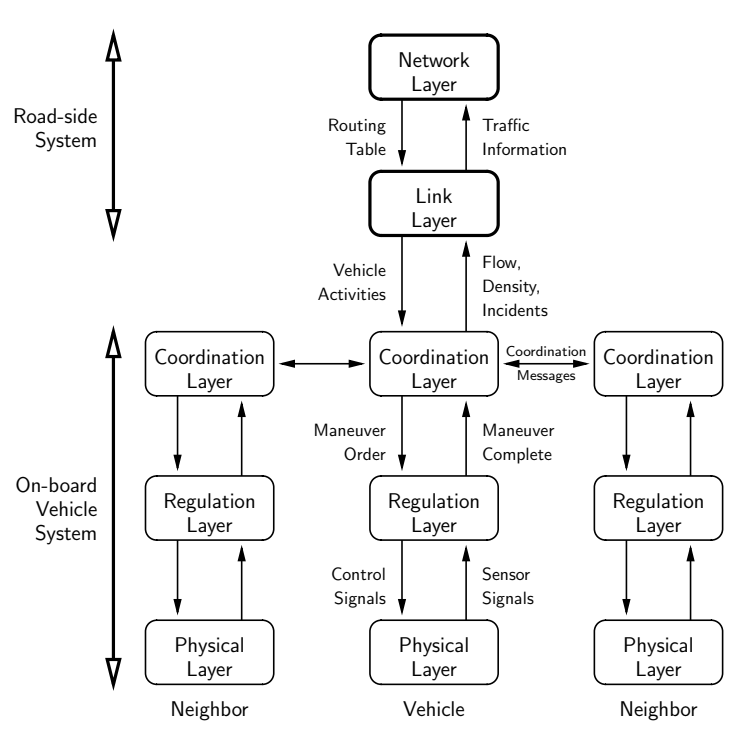

Figure 1. The control system hierarchy in the California PATH AHS architecture.

network layer models the highway as a capacitated graph and its main functions are to control entering traffic and to route traffic flow within an AHS network. A link layer controller computes and broadcasts an activity plan, i.e., the route, maneuvers to be executed, speed, platoon size, etc., for each vehicle type in the link, which is a $0.5-5-\mathrm{km}$-long highway segment, based on a fluid flow model with distributed controls.

The other three layers reside on board an automated vehi- 
cle and their primary objective is to safely control the vehicle while executing its activity plan. The coordination layer communicates and coordinates with peer vehicles and selects one maneuver to be executed. The regulation layer controller is responsible for longitudinal and lateral guidance of the vehicle and execution of the maneuver commanded by the coordination layer. It accepts the sensed and communicated information, such as the velocities and accelerations of itself and neighboring vehicles, from the physical layer and sends the control commands, such as steering and acceleration/deceleration, to the physical layer, which includes all the controllers for the physical components such as the engine, the transmission, brake and steering systems, the guidance and range sensors, and the inter-vehicle radio communication system. The physical layer decouples and linearizes the longitudinal and lateral vehicle dynamics.

The activity plan determined by the link layer controller is consisted of atomic maneuvers from a predefined finite set, which includes lead, follow, split, join, lane-change, etc., with the following restrictions:

1. Only leaders (and free agents) ${ }^{1}$ can initiate maneuvers, while followers maintain platoon formation at all time.

2. Leaders can only execute one maneuver at a time.

3. Maneuvers are coordinated between the leaders of neighboring platoons and a maneuver is initiated only after an agreement is reached between these leaders.

These restrictions ensure safety and significantly reduce the complexity of the link and coordination layer tasks. However, they also cause inefficiency in highway capacity in many cases. For example, when a follower $c$ in a platoon $A$ is to change lanes and join another platoon $B$ on the adjacent lane, in addition to the lane change itself, 3 split and 3 join maneuvers have to executed in the worst case: first, $c$ and followers behind $c$ split from the platoon; then the followers behind $c$ split from $c$ thus $c$ becomes a free agent; in the mean time, the platoon $B$ splits into 2 platoons and opens up a desired space; after that, $c$ changes lanes; then $c$ merges with the first part of platoon $B$ and then the second part merges with the first part; in the mean time, the second part of platoon $A$ merges with the first part and maneuvers are completed.

The inefficiency is in both the space and time. The designed inter-platoon space, which is usually about $60 \mathrm{~m}$, is much larger than the intra-platoon space, which is 1-2 m. On the other hand, it takes much longer time to execute a split or join maneuver than a lane-change. We measure the efficiency of a maneuver by the space-time that it occupies, which is defined as the integral of the space over time. As we mentioned earlier, in the worst scenario, a lane-change procedure requires 3 split and 3 join maneuvers plus the lane-change itself. A split or join maneuver, from 1-m intra-platoon spacing to 60-m inter-platoon spac-

\footnotetext{
${ }^{1}$ The following naming convention is used throughout this paper: the lead vehicle of a platoon is called the leader; the other platoon member vehicles are called the followers; a one-vehicle platoon is called a free agent.
}

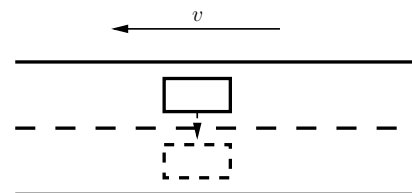

(a) Free agent to free agent lane change.

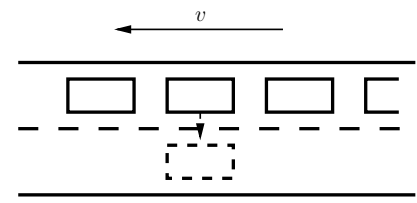

(c) Platoon to free agent lane change. (b) Free agent to platoon lane change.

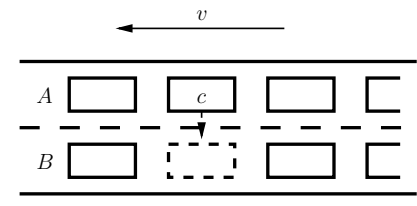

(d) Platoon to platoon lane change.

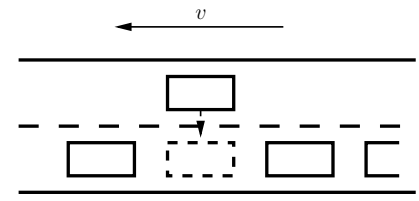

Figure 2. The four scenarios for the lane change within platoons maneuver.

ing, usually takes about $15 \mathrm{~s}$ to complete at a velocity of about $25 \mathrm{~m} / \mathrm{s}$ [3], while a lane-change takes about 5 s [4]. For simplicity, we assume that the spacing changes linearly with respect to time during a split or join. Therefore, without considering the coordination delay, etc., we estimate that the total space-time the lane-change procedure occupies is approximately $4470 \mathrm{~m} \cdot \mathrm{s}$.

\section{LANE CHANGING WITHIN PLATOONS}

To improve the capacity of an automated highway, we propose to eliminate the restriction that only a free agent can change lanes. Instead, a follower can also change lanes without splitting from the platoon first. The follower send a request for lane change to its leader, which coordinates the request with other leaders in the vicinity and decides to approve or disapprove the request depending on whether an agreement is reached between the neighboring leaders or not.

Furthermore, we propose to eliminate the restriction that any vehicle can only be engaged in one activity ${ }^{2}$ at a time. More specifically, we propose to allow a vehicle to be engaged in both following and lane changing activities at the same time. This is justified by the fact that following is a longitudinal task, while lane changing is a lateral maneuver, and the longitudinal and lateral vehicle dynamics are decoupled as a part of the physical layer design.

We propose to include a maneuver called lane change within platoons, which allows a member (either the leader or a follower) of a platoon of more than one vehicle to change lanes without first splitting from the platoon and becoming a free agent. As illustrated in Figure 2, based on different scenarios, this maneuver is sub-classified into 4 cases: the original free-agent-to-freeagent lane change, free-agent-to-platoon lane change, platoonto-free-agent lane change, and platoon-to-platoon lane change.

Among these cases, the platoon-to-platoon lane change is

\footnotetext{
${ }^{2}$ All longitudinal and lateral tasks and maneuvers, such as leading, following, splitting, joining, lane keeping, lane changing, etc., are referred to as activities.
} 


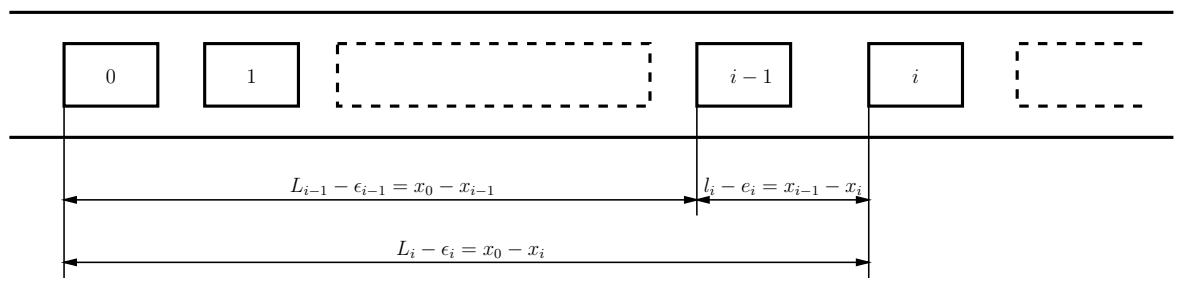

Figure 3. A one-lane platoon of automated vehicles.

Table 1. Comparison of estimated time and space-time needed by the original follower lane-change and the proposed lane-change-withinplatoons maneuvers.

\begin{tabular}{l||c|c}
\hline \hline Maneuvers & Time $(\mathrm{s})$ & Space-time $(\mathrm{m} \cdot \mathrm{s})$ \\
\hline \hline Original lane change & 35 & 4470 \\
\hline Proposed lane change & 11 & 128 \\
\hline \hline
\end{tabular}

the most complicated and the most general one. The other cases can be treated as special cases of the platoon-to-platoon lane change. Thus in the following design and analysis, we only consider this case and the results can be easily applied to the other cases.

In a platoon-to-platoon lane change, the two platoons $A$ and $B$ first align themselves to each other properly and lock their longitudinal positions after they agree to engage themselves in the maneuver, as shown in Figure 2(d). Then the spacings in front of and behind the lane changing vehicle $c$ in the origin platoon $A$ are increased, and a proper space is opened up in the target platoon $B$. After that, $c$ moves laterally into the vacant space in platoon $B$. Upon finishing the lateral movement, the two locked platoons are dissolved. And to finish the maneuver, the increased intraplatoon spacings are reduced to the original values. Now the two platoons are ready for the next maneuver in their activity plans.

We also estimate the space-time occupied by the lanechange-within-platoons maneuver. We assume that in the origin platoon the spacings in front of and behind the lane changing vehicle are increased from $1 \mathrm{~m}$ to $2 \mathrm{~m}$, one after another, which takes about $3 \mathrm{~s}$ each, while at the same time in the target platoon, a space of $9 \mathrm{~m}$ is opened from $1 \mathrm{~m}$, which takes about $6 \mathrm{~s}$, according to the trajectory design we will discuss later in this paper. We also assume that the lateral movement for the vehicle takes $5 \mathrm{~s}$. The approximate space-time that the maneuver occupies is only $128 \mathrm{~m} \cdot \mathrm{s}$.

We summarize the efficiency gain in terms of maneuver time and space-time for the lane-change-within-platoons maneuver in Table 1. As can be seen, the improvement in maneuver efficiency and highway utilization is significant. ${ }^{3}$

\footnotetext{
${ }^{3}$ It is worthy to note that in these estimates of occupied space-time by the
}

\section{STRING STABILITY AND LONGITUDINAL CONTROL Platoon string Stability}

For an inter-connected system, such as a platoon of automated vehicles, stability of each component system itself is not sufficient to guarantee a certain level of performance, such as the boundedness of the spacing errors for all the vehicles. In addition, another stability criterion known as the string stability [5] is needed.

Given a platoon, as shown in Figure 3, we denote the leader by 0 , and number the followers $1,2, \ldots$ from the leader. For a follower $i$, its desired position, measured from the leader 0 , is $L_{i}$. We define its position error

$$
\epsilon_{i}:=x_{i}-x_{0}+L_{i},
$$

where $x_{0}$ and $x_{i}$ is the position of the leader and the follower $i$ relative to a fixed coordinate system. Similarly, we denote the desired spacing, measured from vehicle $i-1$ to $i$, by $l_{i}$, and define the spacing error

$$
e_{i}:=x_{i}-x_{i-1}+l_{i}
$$

Note that $\epsilon_{1}=e_{1}$.

Definition 1 (String stability of a platoon [6]) A platoon is string stable if $\forall \gamma>0, \exists \delta>0$ such that

$$
\max \left\{\sup _{i}\left|e_{i}(0)\right|, \sup _{i}\left|\dot{e}_{i}(0)\right|, \sup _{i}\left|\epsilon_{i}(0)\right|, \sup _{i}\left|\dot{\epsilon}_{i}(0)\right|\right\}<\delta
$$

implies

$$
\sup _{i}\left\|e_{i}\right\|_{\infty}<\gamma
$$

where $\left\|e_{i}\right\|_{\infty}=\sup _{t}\left|e_{i}(t)\right|$.

maneuvers, the coordination and communication delays are neglected. In both the original and proposed lane-change maneuvers, coordination and communication are essential to ensure safety. The proposed lane-change-within-platoons maneuver may require more complicated coordination protocols and thus result in larger delays, because of the more complex maneuver procedure and smaller safety margin. Therefore, the space-time occupied due to coordination and communication delays is larger for the lane-change-within-platoons than for the original maneuver. This might reduce the efficiency gain as estimated in Table 1. However, we still expect this efficiency gain to be significant. 


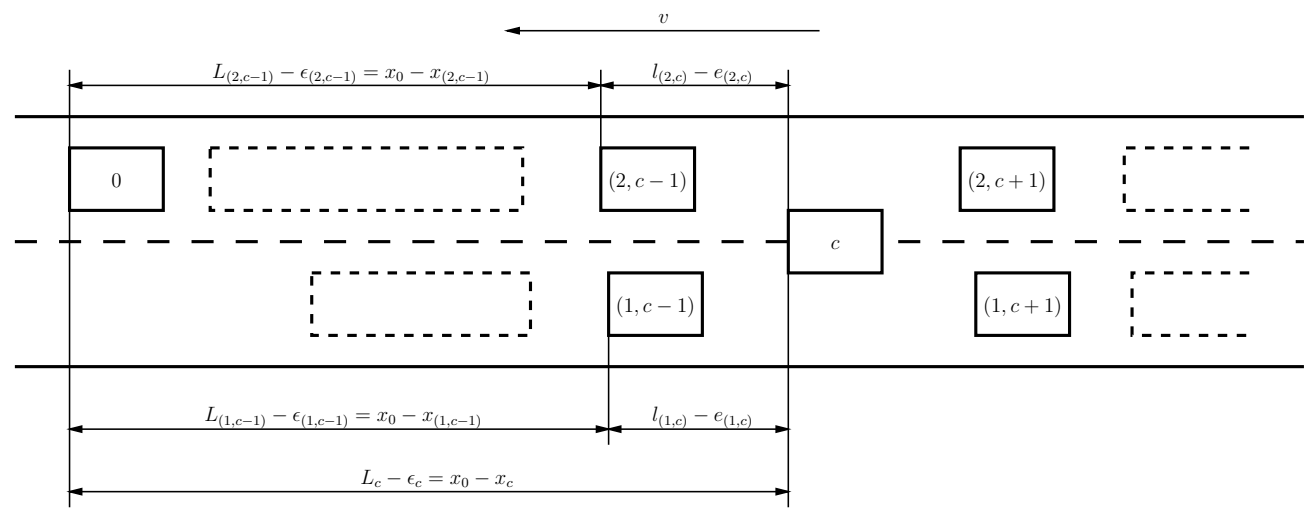

Figure 4. Two locked platoons for lane changing. In the figure, the follower $c$ is carrying out the lane change within platoon maneuver.

In the definition, $\dot{e}_{i}$ and $\dot{\epsilon}_{i}$ are the velocity errors with respect to the preceding vehicle and the leader, respectively.

A sufficient condition [6] for the string stability is that with zero initial errors,

$$
\left\|e_{i}\right\|_{\infty}<\left\|e_{i-1}\right\|_{\infty}
$$

is true for all $i$. If we define the transfer function from $e_{i-1}$ to $e_{i}$ to be

$$
G(s)=\frac{\hat{e}_{i}(s)}{\hat{e}_{i-1}(s)},
$$

where $\hat{e}_{i}(s)$ is the Laplace transform of $e_{i}(t)$, and let $g(t)$ be the impulse response of $G(s)$, then condition (5) for the string stability becomes

$$
\|g(t)\|_{1}<1,
$$

due to a well-known result in system theory that

$$
\left\|e_{i}\right\|_{\infty} \leq\|g(t)\|_{1}\left\|e_{i-1}\right\|_{\infty}
$$

Remark 1 (Necessity of (5)) Note that neither (5) nor (7) is a necessary condition for the sting stability, since (4) only requires that the $\mathcal{L}_{\infty}$-norms of the spacing errors are uniformly bounded with respect to the vehicle index $i$. Therefore, we can allow a finite number of spacing errors to grow by a finite factor, i.e.,

$$
\left\|e_{k}\right\|_{\infty}<M\left\|e_{k-1}\right\|_{\infty}, \quad \text { for } k \in K
$$

where $1<M \in \mathbb{R}$ is a finite gain, and $K$ is a finite set of the vehicle indices. We will use this observation later in the analysis of the longitudinal control laws for the lane change within platoons maneuver.

\section{Longitudinal Control for the Lane Changing Vehicle}

As we described earlier, before a vehicle can change lanes within the platoons, the two platoons involved in the maneuver must align and lock their longitudinal positions with each other and follow a common leader. Therefore, they in effect become one platoon that occupies two adjacent lanes. In the remaining part of this section, we will design a longitudinal control law for the followers of the locked platoons and prove that this follower law is string stable according to Definition 1 . We will discuss the leader law for the common leader in the next section.

Figure 4 shows a schematic of two locked platoons. In the figure, similar to a one-lane platoon, we denote the common leader by 0 . A follower is in the middle of lane changing and it is denoted by $c$. Each of the other followers is denoted by a 2-tuple $(i, k)$, where $i \in\{1,2\}$ denotes the platoon that the vehicle is in, and $k \in \mathbb{N}$ denotes the position where the vehicle is within platoon $i$.

In [6], the follower law is designed using a control surface $S=0$. The surface is chosen so that when the vehicle dynamics stays on $S=0$, the maximum absolute spacing error decays along the platoon in the upstream direction. And the actual vehicle control command $u$, which is the acceleration of the vehicle, is computed so that $S$ converges to 0 exponentially, i.e., the dynamics of $S$ are

$$
\dot{S}+\lambda S=0 .
$$

We will follow a similar approach in our follower law design.

We consider a control strategy that utilizes both the leader and the preceding vehicle position, velocity and acceleration information. For each of the followers other than the one that is changing lane, we define the same control surface $S$ as the one in [6]:

$$
S_{(i, k)}=\dot{e}_{(i, k)}+a_{1} e_{(i, k)}+a_{2} \dot{\epsilon}_{(i, k)}+a_{3} \epsilon_{(i, k)}, \text { for }(i, k) \neq c .
$$

Swaroop has shown [6] that when

$$
a_{1} a_{2} \geq a_{3},
$$


the spacing errors satisfy the following relation:

$$
\left\|e_{i}\right\|_{\infty} \leq \frac{a_{1}}{a_{1}+a_{3}}\left\|e_{i-1}\right\|_{\infty} .
$$

Applying this result to the followers in each of the two platoons, we have

$$
\left\|e_{(i, k)}\right\|_{\infty} \leq \frac{a_{1}}{a_{1}+a_{3}}\left\|e_{(i, k-1)}\right\|_{\infty}, \text { for } i=1,2 \text { and }(i, k)<c,
$$

i.e., the maximum absolute spacing errors decrease along the platoon up to the one preceding the lane changing vehicle $c$.

For the lane changing vehicle $c$ to avoid collision to either of its preceding vehicles in the two platoons, $(1, c-1)$ and $(2, c-$ $1)$, the longitudinal control law needs to ensure that both of the spacing errors, $e_{(1, c)}$ and $e_{(2, c)}$, as shown in Figure 4, are bounded appropriately.

We define a combined spacing error

$$
e_{c}:=\frac{1}{2}\left(e_{(1, c)}+e_{(2, c)}\right)
$$

and the control surface for the lane changing vehicle $c$

$$
S_{c}=\dot{e}_{c}+a_{1} e_{c}+a_{2} \dot{\epsilon}_{c}+a_{3} \epsilon_{c}
$$

We need to find out how the spacing errors propagate from vehicles $(1, c-1)$ and $(2, c-1)$ to $c$ when the vehicle dynamics stay on the $S=0$ surface.

Noticing $e_{(i, k)}=\epsilon_{(i, k)}-\epsilon_{(i, k-1)}$, we have

$$
\begin{aligned}
S_{c}-S_{(1, c-1)} \\
=\dot{e}_{c}+a_{1} e_{c}+a_{2} \dot{e}_{(1, c)}+a_{3} e_{(1, c)}-\dot{e}_{(1, c-1)}-a_{1} e_{(1, c-1)} \\
=\frac{1}{2}\left[\left(1+2 a_{2}\right) \dot{e}_{(1, c)}+\dot{e}_{(2, c)}+\left(a_{1}+2 a_{3}\right) e_{(1, c)}+a_{1} e_{(2, c)}\right] \\
\quad-\dot{e}_{(1, c-1)}-a_{1} e_{(1, c-1)} \\
=0
\end{aligned}
$$

By taking Laplace transform on both sides of (16), we have

$$
\frac{1}{2}\left[\left(1+2 a_{2}\right) s+\left(a_{1}+2 a_{3}\right)\right] \hat{e}_{(1, c)}+\frac{1}{2}\left(s+a_{1}\right) \hat{e}_{(2, c)}=\left(s+a_{1}\right) \hat{e}_{(1, c-1)} .
$$

Similarly,

$$
\frac{1}{2}\left(s+a_{1}\right) \hat{e}_{(1, c)}+\frac{1}{2}\left[\left(1+2 a_{2}\right) s+\left(a_{1}+2 a_{3}\right)\right] \hat{e}_{(2, c)}=\left(s+a_{1}\right) \hat{e}_{(2, c-1)} .
$$

Write (17) and (18) in matrix form and we obtain

$$
\begin{aligned}
& \frac{1}{2}\left[\begin{array}{cc}
\left(1+2 a_{2}\right) s+\left(a_{1}+2 a_{3}\right) & s+a_{1} \\
s+a_{1} & \left(1+2 a_{2}\right) s+\left(a_{1}+2 a_{3}\right)
\end{array}\right]\left[\begin{array}{l}
\hat{e}_{(1, c)} \\
\hat{e}_{(2, c)}
\end{array}\right] \\
& =\left(s+a_{1}\right)\left[\begin{array}{l}
\hat{e}_{(1, c-1)} \\
\hat{e}_{(2, c-1)}
\end{array}\right] .
\end{aligned}
$$

For simplicity of the notations, we define $A=\left(1+2 a_{2}\right) s+\left(a_{1}+\right.$ $2 a_{3}$ ) and $B=s+a_{1}$, and (19) becomes

$$
\frac{1}{2}\left[\begin{array}{ll}
A & B \\
B & A
\end{array}\right]\left[\begin{array}{l}
\hat{e}_{(1, c)} \\
\hat{e}_{(2, c)}
\end{array}\right]=B\left[\begin{array}{l}
\hat{e}_{(1, c-1)} \\
\hat{e}_{(2, c-1)}
\end{array}\right]
$$

Solve these equations and we have

$$
\left[\begin{array}{l}
\hat{e}_{(1, c)} \\
\hat{e}_{(2, c)}
\end{array}\right]=\frac{2 B}{A^{2}-B^{2}}\left[\begin{array}{cc}
A & -B \\
-B & A
\end{array}\right]\left[\begin{array}{l}
\hat{e}_{(1, c-1)} \\
\hat{e}_{(2, c-1)}
\end{array}\right]
$$

We define a coordinate transformation matrix $T=\left[\begin{array}{cc}\frac{1}{2} & \frac{1}{2} \\ \frac{1}{2} & -\frac{1}{2}\end{array}\right]$ and $T^{-1}=\left[\begin{array}{cc}1 & 1 \\ 1 & -1\end{array}\right]$ and left multiply $T$ to both sides of (21)

$$
\begin{aligned}
& {\left[\begin{array}{l}
\frac{e_{(1, c)}+e_{(2, c)}}{2} \\
\frac{e_{(1, c)}-e_{(2, c)}}{2}
\end{array}\right]=\left[\begin{array}{cc}
\frac{2 B}{A+B} & 0 \\
0 & \frac{2 B}{A-B}
\end{array}\right]\left[\begin{array}{l}
\frac{e_{(1, c-1)}+e_{(2, c-1)}}{2} \\
\frac{e_{(1, c-1)}-e_{(2, c-1)}}{2}
\end{array}\right]} \\
& =\left[\begin{array}{cc}
\frac{s+a_{1}}{\left(1+a_{2}\right) s+\left(a_{1}+a_{3}\right)} & 0 \\
0 & \frac{s+a_{1}}{a_{2} s+a_{3}}
\end{array}\right]\left[\begin{array}{c}
\frac{e_{(1, c-1)}+e_{(2, c-1)}}{2} \\
\frac{e_{(1, c-1)}-e_{(2, c-1)}}{2}
\end{array}\right] \\
& =\left[\begin{array}{cc}
H_{1}(s) & 0 \\
0 & H_{2}(s)
\end{array}\right]\left[\begin{array}{l}
\frac{e_{(1, c-1)}+e_{(2, c-1)}}{2} \\
\frac{e_{(1, c-1)}-e_{(2, c-1)}}{2}
\end{array}\right] \text {, }
\end{aligned}
$$

where $H_{1}(s)=\frac{s+a_{1}}{\left(1+a_{2}\right) s+\left(a_{1}+a_{3}\right)}$ and $H_{2}(s)=\frac{s+a_{1}}{a_{2} s+a_{3}}$.

It is a well known result in system theory that

$$
\|h(t)\|_{1} \geq\|H(s)\|_{\infty} \geq|H(0)|
$$

where $h(t)$ is the impulse response of $H(s)$. When $h(t)$ does not change sign for all $t$, the equalities are attained.

Let $h_{1}(t)$ and $h_{2}(t)$ be the impulse responses of $H_{1}(s)$ and $\mathrm{H}_{2}(\mathrm{~S})$, respectively. When

$$
a_{1} a_{2} \geq a_{3}
$$

$h_{1}(t) \geq 0$ and $h_{2}(t) \geq 0$ for all $t$. Note here that (24) is the same condition as (11).

Under this condition,

$$
\left\|h_{1}(t)\right\|_{1}=\left|H_{1}(0)\right|=\frac{a_{1}}{a_{1}+a_{3}}<1,
$$

and

$$
\left\|h_{2}(t)\right\|_{1}=\left|H_{2}(0)\right|=\frac{a_{1}}{a_{3}}<1,
$$

if

$$
a_{3}>a_{1} \text {. }
$$


Therefore, we have

$$
\left\|e_{c}\right\|_{\infty}=\left\|\frac{e_{(1, c)}+e_{(2, c)}}{2}\right\|_{\infty}<\left\|\frac{e_{(1, c-1)}+e_{(2, c-1)}}{2}\right\|_{\infty},
$$

and

$$
\left\|\frac{e_{(1, c)}-e_{(2, c)}}{2}\right\|_{\infty}<\left\|\frac{e_{(1, c-1)}-e_{(2, c-1)}}{2}\right\|_{\infty} .
$$

Therefore

$$
\begin{aligned}
\left\|e_{(1, c)}\right\|_{\infty} & \leq\left\|\frac{e_{(1, c)}+e_{(2, c)}}{2}\right\|_{\infty}+\left\|\frac{e_{(1, c)}-e_{(2, c)}}{2}\right\|_{\infty} \\
& <\left\|\frac{e_{(1, c-1)}+e_{(2, c-1)}}{2}\right\|_{\infty}+\left\|\frac{e_{(1, c-1)}-e_{(2, c-1)}}{2}\right\|_{\infty} \\
& \leq\left\|e_{(1, c-1)}\right\|_{\infty}+\left\|e_{(2, c-1)}\right\|_{\infty} .
\end{aligned}
$$

Similarly,

$$
\left\|e_{(2, c)}\right\|_{\infty}<\left\|e_{(1, c-1)}\right\|_{\infty}+\left\|e_{(2, c-1)}\right\|_{\infty} .
$$

For the two vehicle immediately following the lane changing vehicle, we define the same control surface as the one described in (10). So for vehicle $(1, c+1)$,

$S_{(1, c+1)}-S_{c}=\left(1+a_{2}\right) \dot{e}_{(1, c+1)}+\left(a_{1}+a_{3}\right) e_{(1, c+1)}-\dot{e}_{c}-a_{1} e_{c}=0$.

Take Laplace transform and we have

$$
\hat{e}_{(1, c+1)}=\frac{s+a_{1}}{\left(1+a_{2}\right) s+\left(a_{1}+a_{3}\right)} \hat{e}_{c}=H_{1}(s) \hat{e}_{c} .
$$

We have established that $\left\|h_{1}(t)\right\|_{1}=\frac{a_{1}}{a_{1}+a_{3}}$ as long as (24) is satisfied. Therefore, $\left\|e_{(1, c+1)}\right\|_{\infty} \leq \frac{a_{1}}{a_{1}+a_{3}}\left\|e_{c}\right\|_{\infty}$. It is obvious that the same result holds for the other immediate follower $(2, c+1)$ and other followers in the two platoons beyond $(1, c+1)$ and $(2, c+1)$.

In summary, we have established that

$$
\begin{gathered}
\left\|e_{(i, k)}\right\|_{\infty} \leq \frac{a_{1}}{a_{1}+a_{3}}\left\|e_{(i, k-1)}\right\|_{\infty}, \text { for } i=1,2 \text { and }(i, k),(i, k-1) \neq c, \\
\left\|e_{c}\right\|_{\infty}<\left\|\frac{e_{(1, c-1)}+e_{(2, c-1)}}{2}\right\|_{\infty}, \\
\left\|e_{(i, c)}\right\|_{\infty}<\left\|e_{(1, c-1)}\right\|_{\infty}+\left\|e_{(2, c-1)}\right\|_{\infty}, \quad \text { for } i=1,2,
\end{gathered}
$$

and

$$
\left\|e_{(i, c+1)}\right\|_{\infty} \leq \frac{a_{1}}{a_{1}+a_{3}}\left\|e_{c}\right\|_{\infty}, \quad \text { for } i=1,2 .
$$

Comparing these results with Definition 1 and Remark 1, we conclude that the locked two platoons are string stable.

Remark 2 According to (30) and (31), it is possible for the spacing errors between the lane changing vehicle $c$ and the vehicles preceding $c$ to increase. To avoid possible collision due to this increase in spacing errors, we can increase the nominal intervehicle space, say by a factor of 2 , using the intra-platoon spacing adjustment that we will discuss later in this paper.
MODIFIED LEADER LAW FOR THE COMMON LEADER

When the leader of the origin platoon receives a lane change request from one of its followers, it coordinates with the leaders of the target platoon and other platoons in the vicinity. If an agreement for lane changing is reached, the leader of the target platoon replies to the leader of the origin platoon with the appropriate position within the target platoon for the lane changing vehicle to insert into. With the knowledge of the lane changing vehicle positions in the origin platoon and in the target platoon, the leader of the origin platoon calculates the relative longitudinal positions for the two platoons. Of the two platoon leaders, the one that is further ahead in the longitudinal position is selected as the common leader for the two locked platoons in the duration of the lane change maneuver. The vehicles in the other platoon adjust their position relative to the common leader using the intra-platoon spacing adjustment procedure discussed in the next section. In this section, we modify the original one-lane platoon leader law for the use of the common leader of the two locked platoons.

For a single lane platoon, Alvarez and Horowitz $[3,7]$ have designed the following leader law based on the so-called safety regions:

$$
\begin{aligned}
& v_{\text {safe }}\left(\Delta x, \Delta \dot{x}, v_{\text {lead }}\right)=-c_{2}-v_{\text {allow }} \\
& \quad+\left[2 a_{\mathrm{min}}^{\text {trail }}(\Delta x+\eta \Delta \dot{x})+\alpha\left(v_{\text {lead }}-v_{\text {allow }}\right)^{2}+a_{\mathrm{min}}^{\text {trail }} c_{2} d\right]^{1 / 2},
\end{aligned}
$$

where $\Delta x$ and $\Delta \dot{x}$ are the distance and relative velocity between the leading and trail platoons, $v_{\text {lead }}$ is the velocity of the leading platoon, $d$ is the braking delay, $\eta>0$ is a gain, $v_{\text {allow }}$ is the allowable relative collision speed, ${ }^{4}$

$$
c_{2}=\left(a_{\max }^{\text {trail }}+a_{\min }^{\text {trail }}\right) d,
$$

and

$$
\alpha=\frac{a_{\mathrm{min}}^{\text {trail }}}{a_{\mathrm{min}}^{\text {lead }}} .
$$

In this design, the accelerating/decelerating capabilities of the leading platoon and the trail platoon are assumed to be within $\left[-a_{\min }^{\text {lead }}, a_{\max }^{\text {lead }}\right]$ and $\left[-a_{\min }^{\text {trail }}, a_{\max }^{\text {trail }}\right]$, respectively.

The desired leader velocity is given by

$$
v_{d}=\min \left\{v_{\mathrm{safe}}, v_{\text {link }}\right\},
$$

where $v_{\text {link }}$ is the velocity given to the leader by the link layer.

In our locked two-lane-platoon situation, we assume that both platoons have the same accelerating/decelerating capabilities, i.e., the same values of $a_{\max }^{\text {trail }}, a_{\min }^{\text {trail }}$ and delay $d$. To avoid

\footnotetext{
${ }^{4}$ If collisions need to be strictly avoided, set $v_{\text {allow }}=0$.
} 
collisions with the leading platoons and obstacles on both lanes, the leader law needs to be aware of the conditions in both lanes. Thus we define

$$
\begin{aligned}
& \Delta x=\min \left\{\Delta x_{1}, \Delta x_{2}\right\}, \\
& \Delta \dot{x}=\min \left\{\Delta \dot{x}_{1}, \Delta \dot{x}_{2}\right\},
\end{aligned}
$$

and

$$
v_{\text {lead }}=\min \left\{v_{\text {lead }, 1}, v_{\text {lead }, 2}\right\}
$$

where the subscripts 1 and 2 refer to the two platoons respectively. Note that $\Delta \dot{x}$ is negative when the velocity of the trail platoon is larger than that of the leading platoon. $\Delta x_{1}, \Delta x_{2}, \Delta \dot{x}_{1}$, $\Delta \dot{x}_{2}, v_{\text {lead, } 1}$, and $v_{\text {lead, } 2}$ are measured and communicated to the selected common leader by the first vehicles (the original leaders) of the two platoons. Using these values in (34) and (37), we obtain the desired velocity of the common leader. This desired velocity is then used as the reference for the leader velocity tracking controller designed in [3].

\section{INTRA-PLATOON SPACING ADJUSTMENT}

In the original design of the AHS, the inter-vehicle space within one platoon, which we refer to as the intra-platoon spacing, is assumed to be constant. However, during different stages of the proposed lane change within platoons maneuver, the intraplatoon spacing needs to be adjusted. For example, one of the two platoons needs to adjust its position relative to the common leader; the space in front of the lane changing vehicle $c$ needs to be increased to accommodate the possibly increased spacing error; a space in the target platoon needs to be opened up for $c$ to move into; and in the final step, these increased spaces have to be closed before the maneuver is completed.

In contrast to the split and join maneuvers, which are done non-operatively between the leading and the trail vehicles, this intra-platoon spacing adjustment is completed using the same follower longitudinal controller, with the full knowledge of the leader and preceding vehicle information. The intra-platoon spacing for follower $i$ is adjusted by changing the desired positions $L_{k}$, for $k \geq i$ and the desired spacing $l_{i}$, along a cubic spline trajectory designed with consideration of the ride comfort.

For ride quality, the vehicle longitudinal acceleration $a$ and its rate of change (jerk) $j$ must be within certain bounds, i.e., $a \in\left[-a_{\mathrm{com}}, a_{\mathrm{com}}\right]$ and $j \in\left[-j_{\mathrm{com}}, j_{\mathrm{com}}\right]$. The commonly accepted comfortable limits for the acceleration and jerk are $a_{\text {com }}=$ $2 \mathrm{~m} / \mathrm{s}^{2}$ and $j_{\text {com }}=2.5 \mathrm{~m} / \mathrm{s}^{3}$, respectively $[8,9]$. We use the same jerk limit in our trajectory design. However, in the process of spacing adjustment, the platoon leader may be engaged in acceleration or deceleration, and the acceleration for the spacing adjustment will be added on top of the leader acceleration. Therefore, we reduce the acceleration limit by half to $1 \mathrm{~m} / \mathrm{s}^{2}$.
In the trajectory design, we assume that the vehicles preceding $i$ are cruising at a constant velocity. ${ }^{5}$ In the following discussion, we take the spacing increase for the follower $i$ as an example and only consider the spacing trajectory $l_{i}(t)$. The position trajectories $L_{k}(t)$ for $k \geq i$ and the cases for the spacing decrease are similar. In this example, we assume that $l_{i}$ is increased from the nominal spacing $l^{0}$, which is $1-2 \mathrm{~m}$ in the current design of AHS, to $l^{0}+\Delta l$, and the time duration is $\left(t_{0}, t_{f}\right]$. The trajectory is divided into 5 stages at time points $t_{1}, t_{2}, t_{3}$, and $t_{4}$. During $\left(t_{0}, t_{1}\right]$, the vehicle increases its acceleration from 0 to $a_{m}$ at a rate of $j_{m}$, where $a_{m}=1 \mathrm{~m} / \mathrm{s}^{2}$ and $j_{m}=2.5 \mathrm{~m} / \mathrm{s}^{3}$. During $\left(t_{1}, t_{2}\right]$, it travels with a constant acceleration of $a_{m}$. Then during $\left(t_{2}, t_{3}\right]$, it decreases its acceleration from $a_{m}$ to $-a_{m}$ at a rate of $-j_{m}$, and then travels with a constant acceleration of $-a_{m}$ during $\left(t_{3}, t_{4}\right]$. In the final stage $\left(t_{4}, t_{f}\right]$, it increases its acceleration from $-a_{m}$ to 0 at a rate of $j_{m}$. ${ }^{6}$

The time points at which the stages are divided are determined by the following formulas:

$$
\begin{aligned}
& t_{1}-t_{0}=\delta t, \\
& t_{2}-t_{1}=\frac{-3 \delta t+\sqrt{\delta t^{2}+4 \Delta l / a_{m}}}{2}, \\
& t_{3}-t_{2}=2 \delta t, \\
& t_{4}-t_{3}=t_{2}-t_{1}, \\
& t_{f}-t_{4}=\delta t,
\end{aligned}
$$

where

$$
\delta t=a_{m} / j_{m}
$$

The desired trajectories are

$$
\begin{gathered}
\ddot{l}_{i}(t)= \begin{cases}j_{m}\left(t-t_{0}\right), & t_{0}<t \leq t_{1}, \\
a_{m}, & t_{1}<t<t_{2}, \\
a_{m}-j_{m}\left(t-t_{2}\right), & t_{2}<t \leq t_{3}, \\
-a_{m}, & t_{3}<t \leq t_{4}, \\
j_{m}\left(t-t_{f}\right), & t_{4}<t \leq t_{f} ;\end{cases} \\
\dot{l}_{i}(t)= \begin{cases}j_{m}\left(t-t_{0}\right)^{2} / 2, & t_{0}<t \leq t_{1}, \\
a_{m}\left(t-t_{1}+\delta t / 2\right), & t_{1}<t \leq t_{2}, \\
a_{m}\left(t-t_{1}+\delta t / 2\right)-j_{m}\left(t-t_{2}\right)^{2} / 2, & t_{2}<t \leq t_{3}, \\
a_{m}\left(t_{4}-t+\delta t / 2\right), & t_{3}<t \leq t_{4}, \\
j_{m}\left(t-t_{f}\right)^{2} / 2, & t_{4}<t \leq t_{f} ;\end{cases}
\end{gathered}
$$

\footnotetext{
${ }^{5}$ This is not true in general, since the leader may be accelerating or decelerating according to the leader law. However, the accelerations of the leader and the preceding vehicle are the known disturbance to the longitudinal controller for the followers and the controller is designed to command the vehicle with an appropriate acceleration according to the desired trajectories and these feedbacks.

${ }^{6}$ Because of the relative small values of $j_{m}$ and $a_{m}$ and the length of $\Delta l$, which is usually more than a couple of meters, there are always 5 stages for the trajectory.
} 

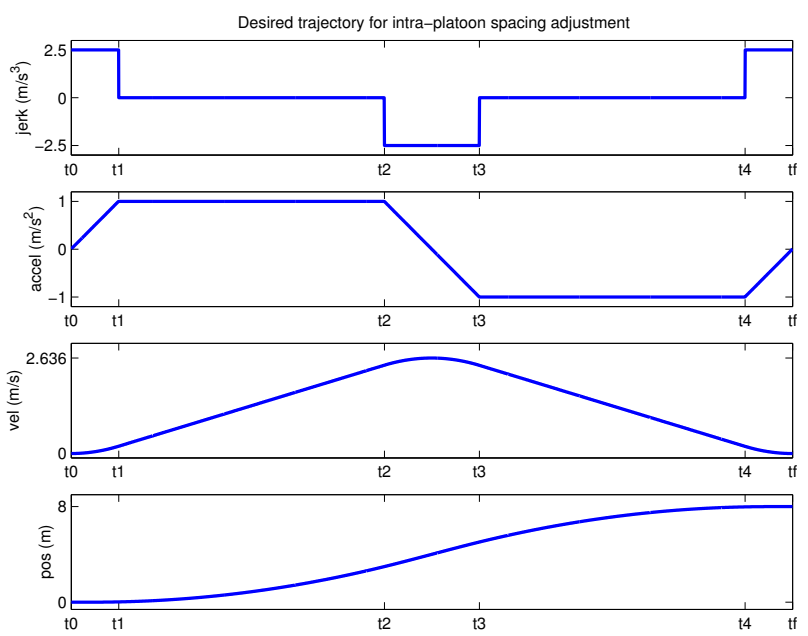

Figure 5. Desired trajectory for intra-platoon spacing adjustment. $\Delta l=$ $8 \mathrm{~m}$ and $t_{f}=6.07 \mathrm{~s}$.

and

$$
l_{i}(t)= \begin{cases}l^{0}, & t \leq t_{0}, \\ l^{0}+j_{m}\left(t-t_{0}\right)^{3} / 6, & t_{0}<t \leq t_{1}, \\ l^{0}+j_{m} \delta t^{3} / 6+a_{m}\left(t-t_{1}\right)^{2} / 2, & t_{1}<t \leq t_{2}, \\ l^{0}+\Delta l / 2+j_{m}\left(t-t_{2}-\delta t\right)^{3} / 6 & t_{2}<t \leq t_{3}, \\ \quad+a_{m}\left(t_{2}-t_{0}\right)\left(t-t_{2}-\delta t\right), & \\ l^{0}+\Delta l-j_{m} \delta t^{3} / 6-a_{m}\left(t-t_{4}\right)^{2} / 2, & t_{3}<t \leq t_{4}, \\ l^{0}+\Delta l+j_{m}\left(t-t_{f}\right)^{3} / 6, & t_{4}<t \leq t_{f}, \\ l^{0}+\Delta l, & t>t_{f} .\end{cases}
$$

Figure 5 shows sample trajectories for a spacing increase of $8 \mathrm{~m}$, which is approximately the space that needs to be opened up in the target platoon for the lane changing vehicle to move into. It takes a little more than 6 seconds to complete this spacing adjustment.

\section{SUMMARY AND FUTURE WORK}

In this paper, we have proposed a new lane-change-withinplatoons maneuver under the California PATH AHS architecture for improving operational efficiency of the current follower-lanechange procedure. The space-time occupied by the lane change procedure decreases from $4470 \mathrm{~m} \cdot \mathrm{s}$ to $128 \mathrm{~m} \cdot \mathrm{s}$, approximately. The designed follower longitudinal control laws are proved to be string stable. The platoon leader law is modified and a new intra-platoon spacing adjustment procedure is designed for the purpose of the proposed maneuver.
The control strategies presented in this paper are currently under implementation by the authors using SmartAHS simulation environment [10]. We are also working on the evaluation and improvement of the performance of the current sensing and communication systems in the AHS architecture for the proposed maneuver. Findings and results will be reported in future publications.

\section{ACKNOWLEDGMENTS}

This work was supported by California PATH (Partners for Advanced Transit and Highways) under Task Order 4239.

\section{REFERENCES}

[1] Varaiya, P., 1993, "Smart Cars on Smart Roads: Problems of Control," IEEE Transactions on Automatic Control, 38(2), pp. 195-207.

[2] Horowitz, R., and Varaiya, P., 2000, "Control Design of an Automated Highway System," Proceedings of the IEEE, 88(7), pp. 913-925.

[3] Alvarez, L., and Horowitz, R., 1999, "Safe Platooning in Automated Highway Systems Part II: Velocity Tracking Controller," Vehicle System Dynamics, 32(1), pp. 57-84.

[4] Peng, H., 1992, "Vehicle Lateral Control for Highway Automation," Ph.D. dissertation, University of California at Berkeley.

[5] Swaroop, D., and Hedrick, J. K., 1996, "String Stability of Interconnected Systems," IEEE Transactions on Automatic Control, 41(3), pp. 349-357.

[6] Swaroop, D. V. A. H. G., 1994, "String Stability of Interconnected Systems: An Application to Platooning in Automated Highway Systems," Ph.D. dissertation, University of California at Berkeley.

[7] Alvarez, L., and Horowitz, R., 1999, "Safe Platooning in Automated Highway Systems Part I: Safety Regions Design," Vehicle System Dynamics, 32(1), pp. 23-55.

[8] Hitchcock, A., 1994, "Casualties in Accidents Occurring During Split and Merge Maneuvers," Technical Memorandum 93-9, California PATH, University of California at Berkeley.

[9] Li, P., Alvarez, L., and Horowitz, R., 1997, “AHS Safe Control Laws for Platoon Leaders," IEEE Transactions on Control Systems Technology, 5(6), pp. 614-628.

[10] Göllü, A., and Varaiya, P., 1998, "SmartAHS: A Simulation Framework for Automated Vehicles and Highway Systems," Mathematical and Computer Modelling, 27(9-11), pp. 103-128. 